\title{
Manifestação rara de edema agudo de pulmão associado à miocardite lúpica aguda - relato de caso
}

\author{
Unusual clinical manifestation of acute pulmonary edema \\ associated to acute lupic myocarditis - case report
}

\author{
Aline Domingos Pinto Ruppert', Marcella Soares Pincelli', Heli Samuel Pinto Souza', \\ Michel Vitor Haddad', Alexandre de Matos Soeiro², \\ Fabrício Sanchez Bergamin ${ }^{3}$, Fernando Ganem ${ }^{4}$
}

Ruppert ADP, Pincelli MS, Souza HSP, Haddad MV, Soeiro AM, Bergamin FS, Ganem F. Manifestação rara de edema agudo de pulmão associado à miocardite lúpica aguda/ Unusual clinical manifestation of acute pulmonary edema associated to acute lupic myocarditis. Rev Med. 2012 abr.-jun.;91(2):83-6.

RESUMO: O lupus eritematoso sistêmico (LES) é a mais comum das doenças auto-imunes sistêmicas. Embora os rins classicamente sejam os órgãos mais acometidos no LES, o coração também pode ser afetado de forma significativa. Entretanto, a ocorrência de edema agudo de pulmão associado à miocardite lúpica é rara e de tratamento imunossupressor específico ainda incerto. $O$ presente relato de caso revisa a literatura quanto a manifestações lúpicas do sistema cardiopulmonar, seu diagnóstico e tratamento, e descreve uma paciente lúpica jovem que evoluiu com edema agudo de pulmão decorrente de uma miopericardite lúpica aguda. O rápido diagnóstico pôde permitir o emprego da terapêutica imunossupressora adequada com reversão completa da disfunção miocárdica. Em pacientes jovens com quadro sugestivo de edema agudo de pulmão, o diagnóstico de LES deve ser considerado. $O$ uso de pulsoterapia com corticóide endovenoso mostrou-se eficaz e seguro para o tratamento da manifestação cardíaca extrema.

DESCRITORES: Lúpus eritematoso sistêmico; Miocardite; Edema pulmonar; Imunossupressores/uso terapêutico.
ABSTRACT: Systemic lupus erythematosus is the most common systemic autoimmune disease. Although kidneys are the main organs affected, heart may suffer injury too. However, acute pulmonary edema associated to lupic myocarditis is rare and its specific immunosuppressive treatment is still undefined. The present case report reviews literature about lupic manifestations in heart and lungs, their diagnosis and treatment, and describes an young lupic patient that had pulmonary edema due to acute lupic myopericarditis. Prompt diagnosis enabled correct immunosuppressive therapy that resulted in a complete reversion of myocardial disfunction. Lupus is a possible diagnosis in young patients with pulmonary edema. The use of intravenous pulse therapy with corticosteroids was safe and efficient to healing of this severe myocardial manifestation.

KEYWORDS: Lupus erythematosus systemic; Myocarditis; Acute pulmonary edema; Immunosuppressive agents/therapeutic use.

Prêmio Oswaldo Cruz - Área Relato de Caso, COMU 2011.

1. Acadêmicos de Medicina, Faculdade de Medicina da Universidade de São Paulo.

2. Orientador, Médico, residência em Clínica Médica e Cardiologia, Instituto do Coração da Faculdade de Medicina da Universidade de São Paulo.

3. Orientador, Médico do Instituto do Coração da Faculdade de Medicina da Universidade de São Paulo.

4. Orientador, Médico, Unidade de Coronariopatia Aguda do Instituto do Coração da Faculdade de Medicina da Universidade de São Paulo.

Endereço para correspondência: Fernando Ganen. Rua Dr. Enéas de Carvalho Aguiar, 44 - Cerqueira César, São Paulo, SP. CEP: 05403-9301 


\section{INTRODUÇÃO} Iupus eritematoso sistêmico (LES) é a
mais comum das doenças auto-imunes sistêmicas, ocorrendo com maior freqüência no sexo feminino, usualmente na faixa etária entre 16 e 55 anos $^{1,2}$. Embora os rins classicamente sejam os órgãos mais acometidos no LES, o coração e a circulação cardiopulmonar também podem ser afetados de forma significativa ${ }^{3}$. Nesse contexto, a ocorrência de edema agudo de pulmão associado à miocardite lúpica é rara e de tratamento imunossupressor específico ainda incerto.

\section{RELATO DO CASO}

Trata-se de paciente de 24 anos, sexo feminino, branca, natural e procedente da cidade de São Paulo, que compareceu ao serviço de emergência com queixa de febre, dor torácica difusa e dispnéia intensa há um dia. Referia quadro prévio de poliartrite aditiva, astenia, alopécia, mialgia e lesão eritematosa em face, tendo permanecido internada em outro serviço com provável diagnóstico de LES há 10 dias. Desde então fazia uso de prednisona $60 \mathrm{mg} / \mathrm{dia}$ e hidroxicloroquina 400mg/dia.

Ao exame físico da admissão encontravase em mal estado geral, taquicárdica (frequência cardíaca = 120 batiemntos por minuto), taquidispneica (frequência respiratória = 32 incursões por minuto), com uso de musculatura acessória, saturação arterial periférica de oxigênio de $87 \%$, pressão arterial de $130 \times 70 \mathrm{mmHg}$, ictus palpável no quarto espaço intercostal sobre a linha hemiclavicular esquerda, presença de bulhas rítmicas com atrito pericárdico audível em mesossistole e estertores finos crepitantes difusos bilateralmente à ausculta pulmonar. Nesse momento foi feito diagnóstico de insuficiência respiratória aguda por provável edema agudo pulmonar, sendo optado por entubação orotraqueal, suporte ventilatório mecânico, administração de furosemida e nitroprussiato de sódio endovenoso.

Solicitado eletrocardiograma que mostrava ritmo sinusal taquicárdico. Radiografia de tórax com cefalização de trama vascular, congestão venosa perihilar, presença de parênquima pulmonar com edema intersticial difuso e área cardíaca com aumento moderado (Figura 1). Ecocardiograma transtorácico mostrou aumento discreto do átrio esquerdo, fração de ejeção de ventrículo esquerdo de $45 \%$ às custas de hipocinesia difusa, insuficiência mitral de grau moderado e derrame pericárdico moderado. Nesse momento foi considerado o diagnóstico de miopericardite aguda secundária ao LES em atividade. Exames laboratoriais corroboraram a hipótese de LES em atividade mostrando leucopenia (3800 leucócitos $/ \mathrm{mm}^{3}$ ), linfopenia (798 linfócitos/ $\mathrm{mm}^{3}$ ), fator antinuclear (FAN) homogêneo > 1:320, anti-DNA positivo, proteína-C reativa de $92 \mathrm{mg} / \mathrm{L}$, velocidade de hemossedimentação de $67 \mathrm{~mm}$ e níveis de complemento (C3 e C4) indetectáveis. Devido hipótese conjunta de atividade lúpica pulmonar, foi solicitado tomografia computadorizada de tórax que mostrou opacidades broncocentricas, pequenas áreas de consolidação pulmonar, derrame pleural e extenso vidro fosco homogêneo bilateral (Figura 2). Como complementação, realizado lavado broncoalveolar que mostrou padrão de hemorragia alveolar discreta, porém com ausência de macrófagos com hemossiderina à microscopia, pouco sugestivo da presença de atividade pulmonar.

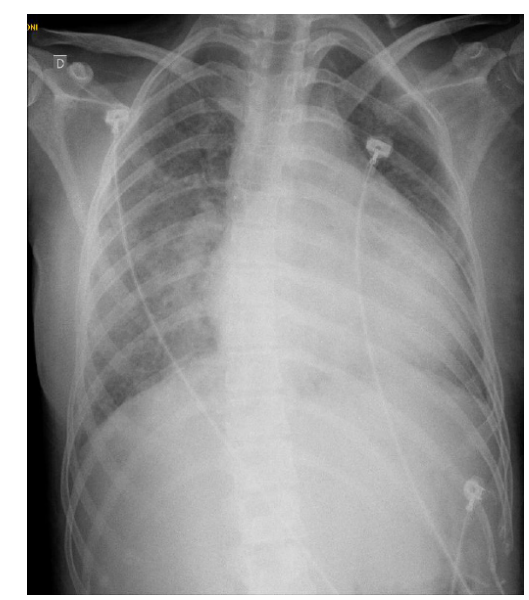

Figura 1. Radiografia de tórax com cefalização de trama vascular, congestão venosa perihilar, presença de parênquima pulmonar com edema intersticial difuso e área cardíaca com aumento moderado

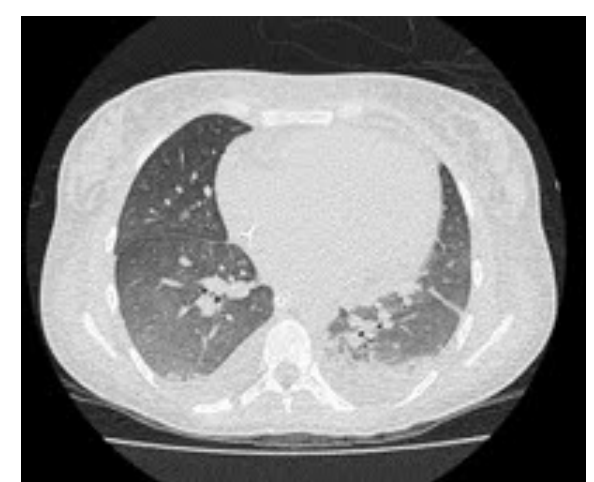

Figura 2. Tomografia computadorizada de tórax que mostrou opacidades broncocêntricas, pequenas áreas de consolidação pulmonar, derrame pleural e extenso vidro fosco homogêneo bilateral

Devido à gravidade do caso associada à disfunção miocárdica aguda, optou-se por iniciar pulsoterapia com metilprednisolona $1 \mathrm{mg} / \mathrm{Kg} /$ dia por três dias consecutivos. Após dois dias a paciente 
apresentou melhora, sendo extubada, em uso de ventilação não-invasiva intermitente, mantendose afebril, hemodinamicamente estável, com desaparecimento da taquicardia mantida, função renal preservada, sem outras intercorrências. A radiografia de tórax apresentava-se sem alterações. Repetido ecocardiograma trans-torácico que evidenciou recuperação importante da função cardíaca (fração de ejeção de ventrículo esquerdo de 56\%) e derrame pericárdico mínimo. Observou-se aumento dos níveis de complemento e foi descartado acometimento renal após realização de proteinúria na urina de 24 horas sem alterações.

A paciente recebeu alta hospitalar assintomática, em uso de prednisona $60 \mathrm{mg} / \mathrm{dia}$, captopril $25 \mathrm{mg} 3 x /$ dia, carvedilol $12,5 \mathrm{mg} 2 x / \mathrm{dia}$, digoxina $0,25 \mathrm{mg} / \mathrm{dia}$, cloroquina $250 \mathrm{mg} / \mathrm{dia}$, ácido fólico $5 \mathrm{mg} /$ dia e sulfametoxazol $800 \mathrm{mg}$ + trimetoprima $160 \mathrm{mg} \mathrm{3x/semana.} \mathrm{Devido} \mathrm{ao} \mathrm{acometimento}$ cardíaco, optou-se por realização de pulsoterapia mensal com ciclofosfamida em nível ambulatorial

\section{DISCUSSÃO}

O diagnóstico de LES foi realizado baseado nos critérios do American College of Rheumatology de 1997 e, portanto, estabelecido através da presença de serosite (derrames pleural e pericárdico), anti-dsDNA positivo, fator antinúcleo positivo e linfopenia.

$\mathrm{Na}$ literatura é descrita a presença de alguma forma de doença cardíaca em 30\% a $89 \%$ dos pacientes com LES ${ }^{1}$. Sua patogênese ainda não está completamente elucidada, sendo atualmente considerada como resultado da deposição de imunocomplexos e ativação do complemento. A agressão pela doença auto-imune pode ocorrer como pericardite, miocardite, endocardite de Libman-Sacks, hipertensão arterial pulmonar e coronariopatia, devendo ser rapidamente reconhecida para estabelecimento de adequada imunossupressão junto à terapêutica cardiológica específica. $O$ tratamento precoce pode contribuir para redução da mortalidade e morbidade na doença, de forma que a cardiopatia no LES é considerada a terceira maior causa de morte, atrás apenas da nefropatia e das infecções ${ }^{1}$.

A pericardite é a forma mais comum de acometimento cardíaco no LES, presente em até $25 \%$ dos pacientes. Geralmente, observam-se pequenos derrames pericárdicos em pacientes assintomáticos em até $54 \%$ dos pacientes ${ }^{2,3}$. Sinais como febre, taquicardia, dor subesternal e atrito pericárdico podem estar presentes, assim como alterações eletrocardiográficas, que de forma geral são indistinguíveis de outras formas de pericardite.
A presença de derrame pericárdico importante e casos de tamponamento cardíaco são $\operatorname{raros}^{3}$. O derrame pericárdico apresenta remissão após o tratamento imunossupressor adequado, assim como no caso apresentando, podendo transformar-se em pericardite constritiva ${ }^{3,4}$.

Referente à miocardite aguda a presença de dor torácica e taquicardia mantida desproporcional à presença de febre pode acorrer assim como relatado 5 . Já sinais e sintomas clínicos de insuficiência cardíaca como no caso apresentado são incomuns, estando presentes em apenas $5 \%$ a $10 \%$ dos pacientes ${ }^{1-3,5}$. Existe enorme dificuldade diagnóstica da miocardite principalmente devido à presença de outros fatores potencialmente responsáveis por dano miocárdico, tais como anemia, hipertensão, infecção e retenção hídrica secundária à doença renal ou ao uso de corticóides ${ }^{1}$. Além disso, miocardiopatia em pacientes com LES pode ter causas distintas como isquemia miocárdica (provocada por arterite coronariana, aterosclerose precoce, trombose ou embolia) e secundária à insuficiência mitral ou aórtica ${ }^{2}$. A presença de disfunção renal poderia retardar o diagnóstico devido à retenção hídrica decorrente. Porém, a ausência de tal manifestação atribui toda a sintomatologia à disfunção miocárdica.

Como descrito anteriormente, a disfunção ventricular decorrente da miocardite lúpica geralmente é de leve intensidade, com sintomas escassos ou ausentes ${ }^{4}$. O ecocardiograma pode mostrar principalmente redução da fração de ejeção (presente em $81 \%$ dos pacientes) e aumento de câmaras cardíacas ${ }^{4,5}$.

Marcadores de atividade inflamatória podem estar elevados em casos de miocardite lúpica. Além disso, os níveis de complemento séricos costumam estar reduzidos, refletindo a atividade da doença. Dentre todos os marcadores, a presença de anti-DNA tem sido associada à miocardite lúpica ${ }^{2}$. Elevação sérica de marcadores de necrose miocárdica como creatinoquinase fração MB (CKMB) e/ou troponina pode ocorrer na miocardite aguda, mas, como em outras etiologias, sua alteração não está presente em todos os casos e não guarda relação direta com a gravidade do quadro clínico ${ }^{2,3}$. Em alguns estudos, anticorpos antimiocárdicos foram observados no sangue de pacientes com LES, porém sua relação com a ocorrência de miocardite aguda ainda é incerta ${ }^{1,2,6}$.

O edema agudo de pulmão associado à miocardite aguda é raro. A boa resposta clínica ao tratamento direcionado como diureticoterapia, uso de vasodilatadores endovenosos e ventilação mecânica não invasiva, associada ao quadro clínico clássico e à imagem observada na radiografia de tórax reforçam 
o diagnóstico de edema agudo de pulmão no caso relatado. Parece haver associação entre congestão venosa pulmonar e derrame pleural à radiografia de tórax em até $73 \%$ dos casos, mesmo não havendo a manifestação de edema agudo pulmonar ${ }^{5}$. Além disso, mesmo a presença de hemorragia alveolar discreta relatada no lavado broncoalveolar também pode por si só ser secundária à congestão venosa pulmonar, uma vez que microscopicamente não se observou a presença de macrófagos com hemossiderina. A atividade lúpica pulmonar é mais incomum do que a atividade cardíaca, e geralmente mesmo após o tratamento adequado costuma deixar algum grau de acometimento permanente significativo principalmente sob a forma de doença pulmonar restritiva, algo que não acorreu nesse $\mathrm{caso}^{2}$. A própria evolução da radiografia de tórax com desaparecimento dos sinais relatados corrobora 0 diagnóstico de edema agudo de pulmão.

O tratamento descrito para casos de disfunção ventricular grave sintomática compreende o uso de doses elevadas de corticóide via oral por 7 a 14 dias, associado à terapêutica cardiológica específica. ${ }^{2,4}$ No caso descrito, devido à gravidade do quadro clínico optou-se por se utilizar pulsoterapia com corticóide endovenoso, associado posteriormente

\section{REFERÊNCIAS}

1. Falcão CA, Lucena N, Alves IC, Pessoa AL, Godoi ET. Cardite lúpica. Arq Bras Cardiol. 2000;74:55-63.

2. Jain D, Halushka MK. Cardiac pathology of systemic lupus erythematosus. J Clin Pathol. 2009;62:584-92.

3. Doria A, laccarino L, Sarzi-Puttini P, Atzeni F, Turriel M, Petri M. Cardiac involvement in systemic lupus erythematosus. Lupus. 2005;14:683-6.

4. Wijetunga M, Rockson S. Myocarditis in systemic lupus erythematosus. Am J Med. 2002;113:419-23.

5. Law WG, Thong BY, Lian TY, Kong KO, Chng $\mathrm{HH}$. Acute lupus myocarditis: clinical features and outcome of an oriental case series. Lupus. 2005;14:827-31.

6. Caforio ALP, Daliento L, Angelini A, Bottaro S, Vinci A, Dequal $\mathrm{G}$, et al. Autoimmune myocarditis and dilated cardiomyopathy: focus on cardiac autoantibodies. Lupus. 2005;14:652-5. à ciclofosfamida. Alguns estudos mostram reversão do acometimento cardíaco com essa abordagem mais agressiva mesmo sem a presença de edema agudo pulmonar ${ }^{2-5,7}$. Não há na literatura estabelecido o que deve ser feito nesses casos, principalmente devido à raridade da manifestação. Obteve-se um bom resultado, inclusive com reversão da disfunção miocárdica observada à ecocardiografia transtorácica. A reversão do dano miocárdico é relatada em até $89 \%$ dos pacientes após seis meses de seguimento, tanto com o uso de corticóide como de imunoglobulina endovenosa ${ }^{7-9}$. Além disso, algumas séries de casos demonstram não haver reincidência do quadro mesmo após quatro anos ${ }^{5,10}$.

\section{CONCLUSÃO}

O presente caso mostra que em pacientes jovens com quadro sugestivo de edema agudo de pulmão, o diagnóstico de LES deve ser considerado. Nessa situação o rápido diagnóstico pode permitir o emprego da terapêutica imunossupressora adequada com conseqüente reversão completa da disfunção miocárdica. Neste paciente o emprego de pulsoterapia com corticóide endovenoso mostrou-se eficaz e seguro para o tratamento da manifestação cardíaca extrema.

7. Chung JW, Joe DY, Park HJ, Kim HA, Park HS, Suh $\mathrm{CH}$. Clinical characteristics of lupus myocarditis in Korea. Rheumatol Int. 2008;28:275-80.

8. Micheloud D, Calderon M, Caparros M, D'Cruz DP. Intravenous immunoglobulin therapy in severe lupus myocarditis: good outcome in three patients. Ann Rheum Dis. 2007;66:986-7.

9. Suri V, Varma S, Joshi K, Malhotra P, Kumari S, Jain S. Lupus myocarditis: marked improvement in cardiac function after intravenous immunoglobulin therapy. Rheumatol Int. 2010;30:1503-5.

10. Apte M, McGwin Jr G, Vilá LM, Kaslow RA, Alarcón GS, Reveille JD. Associated factors and impact of myocarditis in patients with SLE from LUMINA, a multiethnic US cohort. Rheumatology. 2008;47:362-7. 